\title{
ANEURISMAS DE LA ARTERIA POPLITEA: REVISIÓN BIBLIOGRÁFICA Y PRESENTACIÓN DE UN CASO.
}

POPLITEAL ARTERY ANEURYSMS: LITERATURE REVIEW AND PRESENTATION OF CASE.

\author{
Rodolfo Raúl Cecenarro ${ }^{1,2}$, José Norberto Allende ${ }^{1}$, Leandro Barreras Molinelli ${ }^{1}$, Facundo Jorge Antueno ${ }^{1}$, \\ Luis Gramática ${ }^{1}$.
}

\section{Resumen:}

Introducción: El aneurisma de la arteria poplítea (AAP) es la dilatación localizada e irreversible de las paredes de la Arteria Poplítea. Es patología poco frecuente, constituye la segunda ubicación más frecuente de aneurismas verdaderos luego de los aórticos, y la primera ubicación dentro de los aneurismas periféricos. Se caracteriza por afectar a principalmente a hombres a una edad promedio de 65 años, cuyas manifestaciones son principalmente de origen isquémico, acabando en amputación del miembro inferior en una frecuencia que oscila según los casos entre un 7 y un 20 por ciento. Nuestro objetivo es realizar la presentación de un caso de AAP con isquemia arterial subaguda, junto con una actualización bibliográfica del tema. Presentación de caso: Se presenta el caso de un paciente de 63 años de edad quien consultó por dolor de miembro inferior 96 horas de evolución, con ausencia de pulsos. Se realizó el diagnóstico de isquemia subaguda evolucionada, no resvascularizable en el contexto de un paciente clínicamente inestable. Se decide amputación de urgencia. En la disección se objetiva la presencia de un AAP. Discusión: Los AAP tienen una incidencia de entre el 0,1 al $1 \%$, presentan clínica variable. La arteriografía es gold standard para su diagnóstico. El tratamiento de elección es quirúrgico, presentándose nuevas opciones terapéuticas. Las variaciones anatómicas de las ramas de la AP son una variable a considerar. Los aneurismas asociados con mayor frecuencia son los poplíteos contralaterales $(57,1 \%)$. Conclusiones: Concluimos que nuestra conducta fue adecuada considerando el estado del paciente.

Palabras clave: aneurisma de la arteria poplítea; reporte de caso; revisión bibliográfica.

\section{Abstract:}

Introduction: The popliteal artery aneurysm (AAP) is localized and irreversible walls of the popliteal artery dilation. It is rare pathology is the second most common location of true aneurysms after aortic, and the first location within the peripheral aneurysms. It is characterized by affecting mainly men at an average age of 65 , whose manifestations are mainly ischemic, finishing lower limb amputation in a frequency ranging as appropriate between 7 and 20 percent. Our goal is to make the presentation of a case of AAP with subacute arterial ischemia, along with a literature review of the topic. Case presentation: the case of a 63 year old who consulted for lower limb pain 96 hours of evolution, with absence of pulses is presented a diagnosis of advanced subacute ischemia was performed resvascularizable not in the context of a clinically unstable patient. emergency amputation is decided. In dissecting the presence of PSA objective. Discussion: The AAP have an incidence of 0.1 to $1 \%$, have variable clinical. Arteriography is the gold standard for diagnosis. The treatment of choice is surgical, presenting new therapeutic options. The anatomical variations of the branches of the PA are variable to consider. Aneurysms are most commonly associated with contralateral popliteal (57.1\%). Conclusions: We conclude that our conduct was appropriate considering the state of the patient.

Keywords: popliteal artery aneurysm; case report; literature review.

1 Servicio de Hemodinamia y Cirugía Vascular, Hospital Nacional de Clínicas, Córdoba, Argentina.

2 Email de contacto: rodolfocecenarro@gmail.com

Recibido: 2016-12-14 Aceptado: 2017-09-01

DOI: 10.31053/1853-0605.v75.n1.16097 


\section{Introducción}

El aneurisma de la arteria poplítea (AAP) es una patología poco frecuente, que aún así constituye la segunda ubicación más frecuente de aneurismas verdaderos luego de los aórticos, y la primera ubicación dentro de los aneurismas periféricos. Se caracteriza por afecta principalmente hombres a una edad promedio de 65 años, siendo sintomático en alrededor del 60 por ciento de los casos, cuyas manifestaciones son principalmente de origen isquémico, acabando en amputación del miembro inferior en una frecuencia que oscila según la literatura entre un 7 y un 20 por ciento ${ }^{(1-6)}$. Se define como aneurisma de la arteria poplítea cuando existe dilatación transversal de la arteria poplítea de más de 2 $\mathrm{cm}$, o mayor a $150 \%$ del diámetro arterial normal ${ }^{(1)}$. La etiología más aceptada es la arteriosclerosis ${ }^{(2)}$, aunque recientemente se han propuesto causas inflamatorias ${ }^{(3 ; 4)}$

El objetivo de este trabajo es realizar la presentación de un caso de aneurisma de arteria poplítea con isquemia arterial subaguda atendido en nuestro servicio en el año 2015 junto con una actualización bibliográfica del tema, realizada en buscadores de internet.

\section{Materiales y métodos}

Se realizó una búsqueda a través de internet utilizando las palabras claves: "aneurisma de arteria poplítea"+ "clínica" + "Epidemiología" + "tratamiento" + "abordajes", tanto en español como en inglés, en los buscadores Pubmed; BVS; UpToDate, Google Académico. El paciente del caso descripto a continuación brindó su consentimiento por escrito para la publicación anónima de información con fines científicos o educativos.

\section{Presentación del caso:}

Se presenta el caso de un paciente de sexo masculino de 63 años de edad, quien consulta por guardia y es atendido por el servicio de Cirugía vascular del Hospital Nacional de Clínicas en el año 2015. El paciente presentaba antecedentes personales patológicos de hipertensión y tabaquismo moderado, antecedentes quirúrgicos de hernioplastia inguinal. Consulta por dolor de miembro inferior derecho de 96hs de evolución, de intensidad 9/10, acompañado de cianosis periférica de dicho miembro, coloración marmórea del mismo y movilidad distal abolida. Ingresa clínicamente inestable, con criterios de síndrome de respuesta inflamatoria generalizada. Se inician medidas de reanimación con volumen. Como hallazgos positivos en laboratorio el paciente presentaba 20.000 glóbulos blancos con fórmula desviada a la izquierda, CPK total de 550, APP de 30\% (ref 50 - 150\%), Plaquetas 50.000 (ref. 150.000 300.000 ), KPTT 56 seg. (ref. 20-39 seg). Se realiza diagnóstico de coagulopatía por consumo. Se inicia reposición de hemoderivados para optimizar coagulación del paciente. Debido a la respuesta parcial al tratamiento médico, una vez mejorada la coagulación, se decide realizar amputación supracondilea de miembro inferior derecho. Durante la cirugía, se diseca rombo poplíteo, observando aneurisma de la arteria poplítea (Imagen $N^{\circ} 1$ ), se finaliza amputación con pase del paciente a unidad de cuidados intensivos. Durante internación se efectúa tomografía computarizada de abdomen con contraste en búsqueda de aneurisma de aorta abdominal, se realizan ecodoppler de vasos de cuello y de miembro inferior izquierdo. En todos los estudios se descarta la presencia de aneurismas concomitantes. El paciente evoluciona favorablemente, se decide alta y control ambulatorio hasta el día de la fecha.

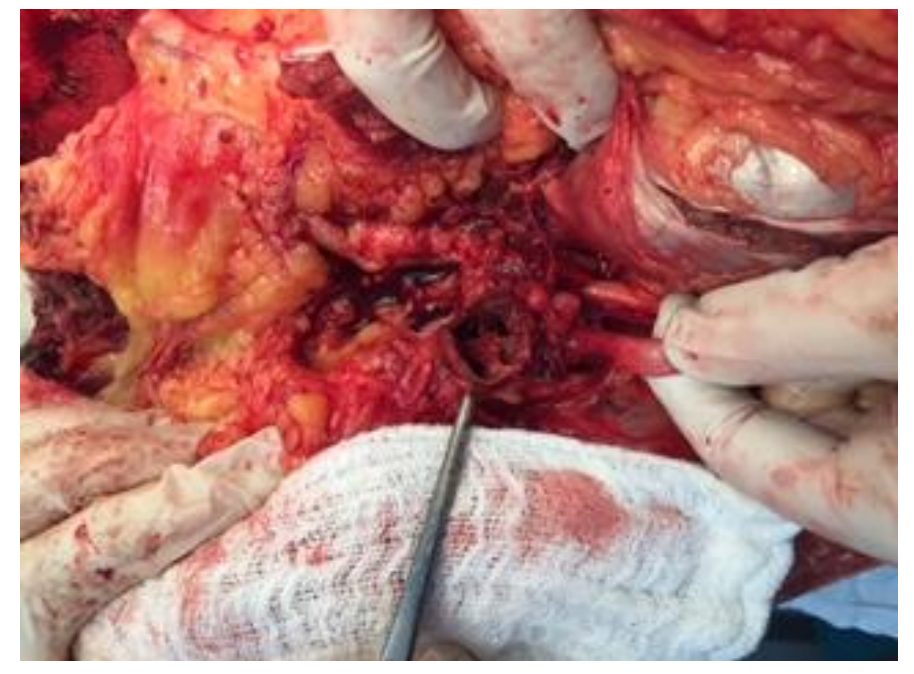




\section{Discusión}

Los aneurismas de la arteria poplítea constituyen un cuadro patológico caracterizado por cuadros clínicos que van desde asintomáticos pasando por isquemias crónicas hasta cuadros de isquemia arterial aguda. La arteria poplítea es la continuación de la arteria femoral superficial, atraviesa la cara posterior de la articulación de la rodilla, en el plano profundo del hueco poplíteo, siendo finalmente la arteria que da origen a los vasos de la pierna y el pie. Nace a nivel del hiato de los aductores, se dirige hacia medial y posterior recorriendo la fosa poplítea terminando en el músculo poplíteo donde se continúa con los vasos tibiales. Sus ramas terminales son la arteria tibial anterior, el tronco tibioperoneo que da origen a las arterias tibial posterior y peronea. Su longitud media varía entre 17 y $18 \mathrm{~cm}^{(5)}$. (Imagen $\left.\mathrm{N}^{\circ} 2\right)$

Su diámetro varía de $0.9 \mathrm{~cm} \pm 0,2 \mathrm{~cm}$, con una longitud total de alrededor de $16 \mathrm{~cm}^{2}$.

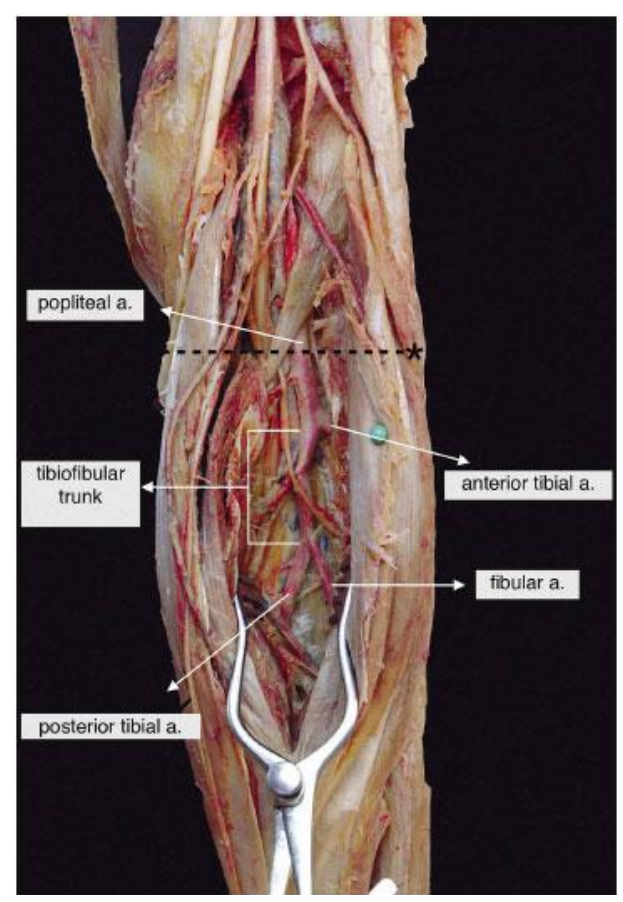

Imagen 2. Relaciones anatómicas de la arteria poplitea

La definición de aneurisma corresponde a una dilatación mayor del $50 \%$ del diámetro del vaso, en el caso de la arteria poplítea, mayor de $1.5 \mathrm{~cm}^{3(6)}$.

La mayoría de los autores coincide en la baja frecuencia de los AAP, pero no dan cifras aproximadas de dicha frecuencia. Para Ticket et al ${ }^{(7)}$, y Hencke et al ${ }^{(8)}$, la frecuencia de AAP en la población general es del $0.1 \%$ ascendiendo al $1 \%$ en hombres de entre 65 y 80 años.

Afectan principalmente a pacientes de sexo masculino entre la sexta y la octava décadas de vida, a una edad promedio de 65 años; presentan una alta tasa de bilateralidad y frecuentemente asociados a aneurismas en otras arterias ${ }^{(1-6)}$.

Los AAP son los más comunes entre los aneurismas periféricos ${ }^{(1 ; 6)}$.

Las complicaciones más frecuente de los AAP son las debidas a trombosis. Un análisis específico de los aneurismas poplíteos trombosados mostró que los dos principales factores de riesgo para predecir trombosis, independientemente de su tamaño y morfología, fueron la hipertensión arterial y la bilateralidad $^{(1)}$. Los mismos autores obtuvieron resultados desfavorables en aquellos pacientes que presentaron complicaciones con cifras de morbilidad de $66.66 \%$, amputación de $33.33 \%$ y mortalidad de $16.66 \%$, en comparación con la nula morbi-mortalidad de los pacientes con aneurisma de la arteria poplítea tratados electivamente.

El $95 \%$ de los pacientes presenta comorbilidades. Merterns et $\mathrm{al}^{(9)}$, evaluaron 33 pacientes seguidos durante 15 años, donde la hipertensión arterial fue la patología asociada de mayor prevalencia, presente en un $71.4 \%$ de los casos, seguida por el tabaquismo (42.9\%) y la enfermedad coronaria (33.3\%). RojasReyna et al ${ }^{(1)}$ tuvo cifras superiores, hallando tabaquismo en el $90.9 \%$ de los pacientes e hipertensión arterial en el $63.6 \%$. En nuestro caso fueron hallados, ambos factores de riesgos, hipertensión y tabaquismo.

Mientras Merterns ${ }^{(9)}$ halló una prevalencia de aneurismas asociados de $57.1 \%$ para los poplíteos bilaterales, $57.1 \%$ para los femorales, $47.6 \%$ para los aórticos y $23.8 \%$ para iliácos, Rojas- Reyna et al ${ }^{(1)}$ $(\mathrm{N}=11)$, obtuvo un $63.63 \%$ que presentaron aneurisma poplíteo bilateral, $54.54 \%$ tenían concomitantemente aneurisma de la aorta abdominal. En nuestro caso, se descartó mediante tomografía computarizada con contraste, la existencia de aneurismas de aorta abdominal. 
La clínica de los AAP es variable, los pacientes pueden presentar síntomas oclusivos crónicos o agudos, por trombosis o embolia, síntomas compresivos del nervio ciático poplíteo o presentarse totalmente asintomáticos en hasta el $40 \%$ de los pacientes ${ }^{(6-14)}$.

Al diagnóstico se llega con la clínica y el uso de métodos complementarios. Existen diferencias marcadas entre distintos autores a la hora de elegir uno como ideal. La ecografía doppler color es una opción válida, sensible, de relativo bajo costo y no invasiva ${ }^{(10)}$. Para otros autores la resonancia magnética y la angioresonancia son opciones incluso superiores a la arteriografía ${ }^{(11)}$, mientras otros como Imigo et al ${ }^{(6)}$ se refieren a la arteriografía como el gold estándar diagnóstico. (Ver imágenes $N^{\circ} 3,4$ y 5)

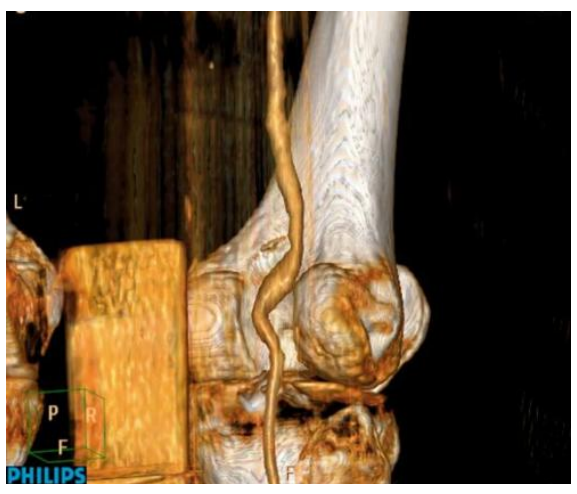

Imagen 3.

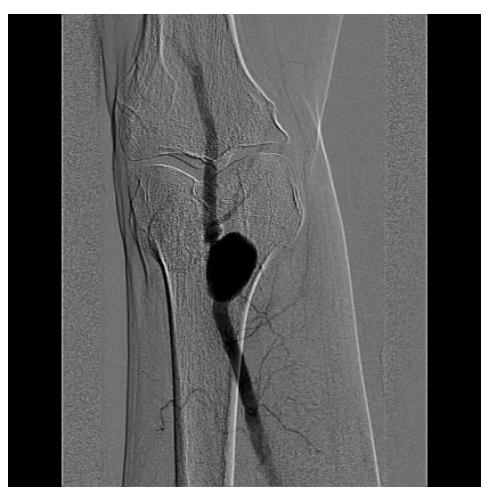

Imagen 4.

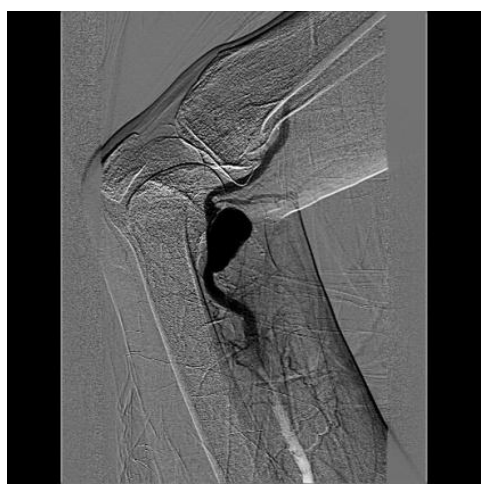

Imagen 5.

La cirugía temprana es la opción terapéutica aún en pacientes asintomáticos ${ }^{(11)}$. Las opciones de tratamiento incluyen la cirugía convencional y la colocación de endoprótesis. Dentro de los procedimientos convencionales, el "gold estándar" consiste en la ligadura proximal y distal de la arteria poplítea por encima y debajo del aneurisma, y el posterior by pass con vena safena autóloga. Existen dos vías de abordaje en cirugía abierta, por vía medial, y posterior; cada una de ellas ofrece ventajas y desventajas. Mazzacaro et al $^{(12)}$, no hallaron diferencias significativas entre ambas vías de abordaje, observandosé buenos resultados para ambas. Hoelting et al ${ }^{(15)}$ describieron el valor de la terapia lítica con urokinasa preoperatoria. En su estudio el índice de amputaciones fue del $9 \%$ para los pacientes tratados previamente con urokinasa, y del $25 \%$ para aquellos que solo fueron tratados con cirugía.

Para Rabellino et al ${ }^{(14)}$ el tratamiento endovascular es una alternativa de primera elección en pacientes con elevado riesgo quirúrgico y condiciones anatómicas favorables. En su trabajo consiguieron un éxito técnico en el $100 \%$ de los casos sin complicaciones durante los 30 días post tratamiento. La media de seguimiento fue de 34 meses, registraron 3 oclusiones, dos de ellas se recanalizaron mediante tratamiento fibrinolítico y la permeabilidad primaria durante el seguimiento fue del $75 \%$ y $92 \%$ la secundaria. Recientemente, en la Universidad de Uppsala, Cervit et al ${ }^{(15)}$ evaluaron 592 pacientes concluyendo que la reparación endovascular (Imagen $N^{\circ} 6$ ), exhibió resultados claramente inferiores a los procedimientos abiertos sobre todo en aquellos pacientes que presentaban isquemia arterial aguda. Similares resultados publicaron Dorigo et al ${ }^{(16)}$, afirmando que a largo plazo la cirugía abierta tiene mejores resultados que los procedimientos endovasculares.

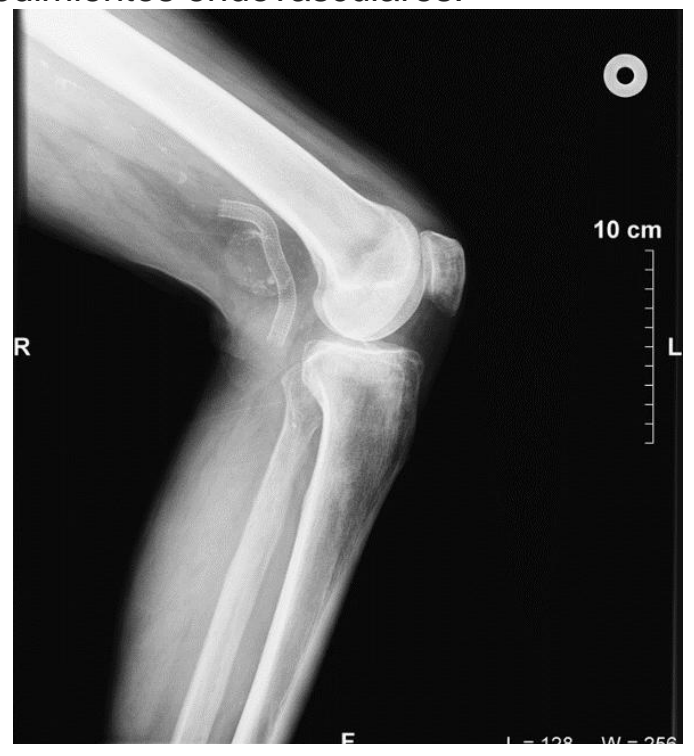

Imagen 6. Reparación endovascular. 
El índice de amputaciones para distintos autores fue variable, ubicándose entre un 7\% y un 33\% con una media cercana al $15 \%{ }^{(8-16)}$.

\section{Conclusión}

Los AAP representan una patología poco común, presente en el $0,1 \%$ de la población general y en hasta el $1 \%$ de los hombres mayores de 65 años. Es importante considerar el rol de las variaciones anatómicas en la presentación, diagnóstico y tratamiento de esta patología.

Se presentan en individuos con numerosos antecedentes personales patológicos, encabezados por la hipertensión arterial y el tabaquismo.

Su clínica es variable; pueden ser asintomáticos o presentarse con isquemias crónicas o agudas.

Sus complicaciones se deben a procesos trombóticos o embólicos principalmente, aunque también producen clínica por compresión periférica.

La conducta quirúrgica debe tomarse en todos los casos, aún en los asíntomáticos, debido al elevado índice de complicaciones.

La ligadura proximal y distal del aneurisma con by pass utilizando safena autóloga, es la conducta de elección. Pueden utilizarse también prótesis para realizar el by pass con buenos resultados. Las vías de abordaje medial y posterior, no presentaron diferencias significativas. El tratamiento prequirúrgico con urokinasa puede mejorar los resultados

El tratamiento endovascular es una opción en aquellos pacientes cuyo riesgo cardiovascular elevado contraindica un procedimiento quirúrgico convencional, aunque se debe tener precaución en isquemias arteriales agudas, donde los procedimientos convencionales ofrecen mejores resultados.

El índice de amputaciones oscila según los autores entre un 7 y un 33\% con una media cercana al 15\%, siendo a veces la única opción.

En relación a la elección de métodos complementarios, las opiniones son variables, la ecografía doppler color y la angioresonancia son buenas opciones, aunque podría decirse que la arteriografía es el gold estándar para el diagnóstico de AAP.

En nuestro caso, consideramos que la conducta tomada fue la adecuada, teniendo en cuenta el grave estado clínico del paciente, que impedía realizar una arteriografía de urgencia, ya que dicho estado era una consecuencia de su isquemia aguda. Si bien el paciente fue amputado, sus parámetros clínicos revirtieron en poco tiempo, sin mortalidad.

\section{Bibliografía}

1. Rojas-Reyna GA, Cervantes-Castro J, Alvarado-Bachmann $R$ y cols. Aneurismas de la arteria poplítea. Treinta años de experiencia en el Centro Médico ABC. Cir Ciruj 2008; 76: 55-59.

2. Szilagyi DE, Schwartz RL, Reddy DJ. Popliteal arterial aneurysms. Their natural history and management. Arch Surg 1981; 116 (5): $724-8$.

3. Jacob T, Ascher E, Hingorani A. Initial steps in the unifying theory of the pathogenesis of artery aneurysms. J Surg Res 2001; 101: 37-43.

4. Jacob T, Schutzer R, Hingorani A. Differential expression of YAMA/CPP-32 by T lymphocytes in popliteal artery aneurysm. $J$ Surg Res 2003; 112: 111-116.

5. Marando Jl, Piuzzi NS, Rousselot Ascarza AM. La arteria Poplitea y sus ramas terminales. Cátedra de Anatomía Normal Luis Dellepiane. Facultad de Medicina. Universidad del Salvador. 2009. Ed. 3, Art 24.

6. Imigo FG, Fonfach CZ, Massri DE y cols. Aneurisma de arteria poplítea. Cuad Cir 2009; 23: $39-43$.

7. Ticket JP, Scout RAP, Tilney HS. Screening and management of asymptomatic popliteal aneurysms. J Med Screen 2002; 9:92-93.

8. Henke PK. Popliteal artery aneurysms: tried, true and new approaches to therapy. Semin Vasc Surg 2005; 18: 224-230.

9. Mertens R, Valdés F, Kramer A. Popliteal aneurysm: results of the management of 33 lesions. Rev Med Chil. 1991; 119(4): 406-11.

10. Weiner SN, Hoffman J, Bernstein RG y cols. The value of ultrasound in the diagnosis of popliteal artery aneurysms. Angiology 1983; 34(6): 418-27.

11. Kolb M, Guhl L, Arlart IP. Magnetic resonance tomography and magnetic resonance angiography in diagnosis of complicated popliteal artery aneurysm. Radiologe 1997; 37(2): 145-51.

12. Mazzaccaro D, Carmo M, Dallatana $R$ y cols. Comparison of posterior and medial approaches for popliteal artery aneurysms. Vasc Surg 2015; 62(6): 1512-20.

13. Hoelting T, Paetz B, Richter GM y cols. The value of preoperative lytic therapy in limb-threatening acute ischemia from popliteal artery aneurysm. Am J Surg 1994; 168(3): 227-31.

14. Rabellino M, González G, Canales E, y cols. Tratamiento endovascular de los aneurismas popliteos. Seguimiento a largo plazo. Intervencionismo 2009; 93: 101-107.

15. Cervin A, Tjärnström J, Ravn $H$ y cols. Treatment of Popliteal Aneurysm by Open and Endovascular Surgery: A Contemporary Study of 592 Procedures in Sweden. J Vasc Endovasc Surg 2015; 50: 342-350.

16. Dorigo W, Pulli R, Alessi Innocenti A y cols. A 33-year experience with surgical management of popliteal artery aneurysms. Journal of Vascular Surgery, 2015; 62: 1176-1182. 